\title{
Papain Activity in Dextran Solution for Keratin Hydrolysis
}

\author{
Nanami Hara, Shintaro Morisada, Keisuke Ohto, Hidetaka Kawakita* \\ Department of Applied Chemistry, Saga University, Saga, Japan \\ Email: ${ }^{*}$ kawakita@cc.saga-u.ac.jp
}

Received 28 January 2014; revised 28 February 2014; accepted 9 March 2014

Copyright (C) 2014 by authors and Scientific Research Publishing Inc.

This work is licensed under the Creative Commons Attribution International License (CC BY). http://creativecommons.org/licenses/by/4.0/

(c) (i) Open Access

\begin{abstract}
High concentration of polymer solution in cosmetics has the essential role in keeping water on peel of skin. This study includes the papain activity in the high concentrated dextran solution for keratin hydrolysis. Papain is the industrial and a representative hydrolase. The concentration of dextran was ranged to $100 \mathrm{~g} / \mathrm{L}$. In the increasing concentration of dextran, the activity of papain decreased due to the low diffusivity of papain and substrate. Even in concentrated dextran solution, keratin powder was possible to be hydrolyzed at the high efficiency.
\end{abstract}

\section{Keywords}

Dextran; Papain; Keratin; Hydrolysis

\section{Introduction}

In chemical peeling, acids such as glycol acid (hydroxyacetic acid) have been applied for removal of old cuticle, reforming to normal peel by natural healing [1]. However, in abrasion of cuticle by acid, excess calcium ion is generated by dissolving protein in cell wall of peel, as a result, removing dead cell. Soluble polymer of cosmetics in considering chemical peeling has the potential requirements of 1) high safety, 2) no color, no odorless, and no taste, 3) high stability and no chemical change, and 4) high solubility to water. In 1), soluble polysaccharide has the high safety against low dermal irritancy and allergy. Water plays the important role in keeping youngness of peel. Also, hydroxyl groups in carboxymethylcellulose [2] and polyvinylalcohol [3] have the unique characteristic to keep water. Hydrogen bonding of hydroxyl group with water is related to maintaining water on peeling. Using this characteristic in cosmetics, the above-mentioned polymers for peeling have high stability to maintain water.

Papain is a kind of hydrolase, extracted from papaya, and has wide substrate specificity, basic amino acid re-

"Corresponding author. 
sidue. The enzyme is largely used for various applications of food engineering [4] and cosmetic [5]. Because papain is produced as an industrial enzyme, it is applicable at low cost for cosmetic compounds. Dextran, a homopolysaccharide of glucose from Leuconostoc mesenteroides with a substantial number of consecutive $\alpha-(1,6)$ linkages, is of commercial interest as a chemical for clinical, pharmaceutical, research, and industrial use [6]. Because the hydroxyl groups on dextran exist at outside of the polymer and dextran forms a random structure, dextran has a higher hydrophilicity and biocompatibility than cellulose. Some researchers have studied dextran for cosmetic [7] and drug engineering to maintain water on peel [8].

The structural organization of the keratin dominated in stratum corneumcorneocyte matrix has major importance for the barrier properties of skin, for the water holding capacity of peel, for the appearance (i.e. optical properties) of peel and for the mechanical strength and elastic resilience of peel. Keratin was composed of seven single polypeptide chains, each with the configuration of a compound $\alpha$-helix, where six such chains twisted about a seventh to form a seven-strand cable [9].

In this study, we have determined the activity of papain against keratin originated from wool in dextran solution. Dextran would function to hydrate the surface of dry keratin via adsorption. The dextran coated on the keratin enhances the deposition of water to hydrolyze easily. Because dextran has plural hydroxyl groups and flexible structure, the structural damage of papain would be reduced. The concentration of dextran is changed from 0 to $100 \mathrm{~g} / \mathrm{L}$ in phosphate buffer solution. During the enzymatic reaction, the viscosity of dextran should be considered because the mobility of the substrate and the enzyme would be limited. The objective of this study was 1) to verify the activity of papain in dextran solution, and 2) to hydrolyze keratin. Papain in dextran solution would work for skin care. Dextran has the high viscosity, resulting in long residence time on skin. This is the first report for the activity of papain in dextran solution aiming for cosmetic engineering.

\section{Experimental}

\subsection{Materials}

Papain and keratin powder were purchased from Wako Pure Chemical Industries, Ltd. Dextran (Mw. 2,000 kDa) was purchased from Sigma-Aldlich Co. (St. Louis, MO). $N$ - $\alpha$-benzoyl-DL-arginine $p$-nitroanilide hydrochloric acid and $p$-nitroanilide were obtained from Sigma-Aldlich Co. (St. Louis, MO). Other chemicals were of analytical grade or higher.

\subsection{Activity of Papain in Dextran Solution}

$\mathrm{N}$ - $\alpha$-benzoyl-DL-arginine $p$-nitroanilide (DL-BAPA) was dissolved to $10 \mathrm{mM}$ of phosphate buffer (pH 7.0) including the various concentrations of dextran (Mw. $2000 \mathrm{kDa}$ ). The concentration of DL-BAPA was $1.0 \mathrm{mM}$. The concentration of papain dissolved in dextran solution was set at $1.0 \mathrm{~g} / \mathrm{L}$. The substrate and the enzyme solutions were mixed to start the reaction at $303 \mathrm{~K}$ at $150 \mathrm{rpm}$ in thermostat shaker. The concentration of $p$-nitroanilide, a product of hydrolyzed DL-BAPA, was determined by UV-VIS (UV 1800, Shimadzu, Japan). The absorbance of $p$-nitroanilide was changed to concentration using Lambert-Beer equation. The activity of papain was determined from the production of $p$-nitroanilide at the initial, as follow,

$$
\text { Enzymatic activity }[\mathrm{mol} / \mathrm{L}[\mathrm{min}]=(\text { the concentration of } p \text {-nitroanilide }) /(\text { reaction time })
$$

The reaction time measured was 60 min after the start of the enzymatic reaction.

The viscosity of dextran solution was measured by DV-II + Pro (VISCOMETER, BROOKFIELD, U.S.A.) at $303 \mathrm{~K}$.

\subsection{Hydrolysis of Keratin by Papain in Dextran Solution}

The concentration of dextran (Mw. $2000 \mathrm{kDa}$ ) was set at $50 \mathrm{~g} / \mathrm{L}$. Papain was added to start the reaction at the concentration of $1 \mathrm{~g} / \mathrm{L}$. The concentration of dry keratin added was $1 \mathrm{~g} / \mathrm{L}$. The temperature was $303 \mathrm{~K}$. In a prescribed time, the reaction solution was filtered using PTFE membrane (Millipore, $0.45 \mu \mathrm{m}$ ) to remove insoluble hydrolyzed keratin. The dissolvable protein or amino acid by hydrolysis in filtrate was determined by UV-VIS (280 nm), changing the absorbance to the concentration. The reaction solution was observed by digital camera. 


\section{Results and Discussion}

\subsection{Papain Activity in Dextran Solution}

Soluble polymer applied for enzymatic reaction is dextran. Dextran has the flexible structure with the plural hydroxyl groups. Water molecule hydrates dextran, resulting in high viscosity with increasing concentration of dextran. Papain is used for industrial enzyme and possible to be obtained at low cost. The activity of papain in dextran solution was determined. Hydrolyzed activity of papain was measured in changing the concentration of dextran (Figure 1). DL-BAPA, a substrate, was hydrolyzed by papain to produce $p$-nitroanilide in a yellow color. As Figure 1, with increasing dextran concentration, the activity of papain gradually decreased.

The activity of papain in dextran solution should be considered based on two follows: 1) diffusion (mobility) of substrate and enzyme, and 2) conformation change of papain by dextran. The mobility of substrate and enzyme is directly related to viscosity of dextran solution. The viscosity of dextran solution buffered by phosphate as a function of dextran concentration is shown in Figure 2. With increasing the concentration of dextran, the viscosity was exponentially increased. This is because the entanglement of dextran via the interactions between hydroxyl groups increased. The mobility or diffusivity of molecules concerns with the viscosity of solution as Einstein-Stokes equation [10]. Diffusivity is limited with the increment of the viscosity. Also the viscosity of dextran reduces the enzymatic activity via fluctuation of enzyme molecule. If the polymer molecule interacts with the enzyme molecule, enzyme structure is changed. However, dextran doe not have the functional group of carboxyl and amino groups. Hydroxyl group in dextran might not destroy the structure of enzyme. It was found that papain had the activity in the high concentrated dextran solution.

\subsection{Keratin Hydrolysis in Dextran Solution}

By removing old cuticle on peel surface, the new dermal tissue would smoothly grow from the inside of skin. Our aim of this paper is the proposal of the removal of old cuticle without using the agent of chemical peeling. Instead of chemicals, the enzymatic reaction in dextran solution is used for peeling by hydrolysis in mild condition. Because old cuticle has low water moisture, for hydrolysis enzyme should be located on the old cuticle with water molecules. Due to its high viscosity dextran would make enzyme locate on the skin for long residence time. Time course curve of keratin hydrolysis with papain in dextran solution is shown in Figure 3. The concentration of hydrolysates after microfiltration in each solution was determined by the absorbance of UV.

In the case of only keratin the concentration of hydrolysates did not change, meaning no occurrence of hydrolysis. Keratin in the presence of papain in the solution was hydrolyzed and the concentration of the hydrolysates reached at equilibrium after 24 hours. Keratin in dextran solution was hydrolyzed by papain, but the hydrolyzed speed was low compared with that in water solution. However, irrespective of high concentration of 50 $\mathrm{g} / \mathrm{L}$ of dextran solution, keratin hydrolysis proceeded. Compared with water solution, the hydrolyzed concentration at the steady state was lower.

The hydrolyzed solutions of keratin were observed in Figure 4. Keratin has the helical structure and the S-S bonding between cysteines. In processing the hydrolysis, cysteins of keratin are cleaved by papain in both water

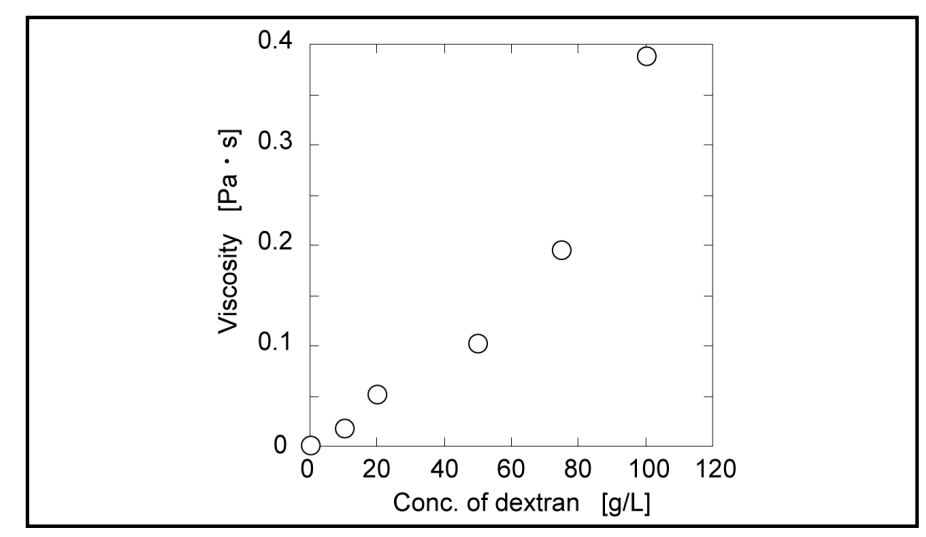

Figure 1. Relationship of activity of papain with concentration of dextran solution. 


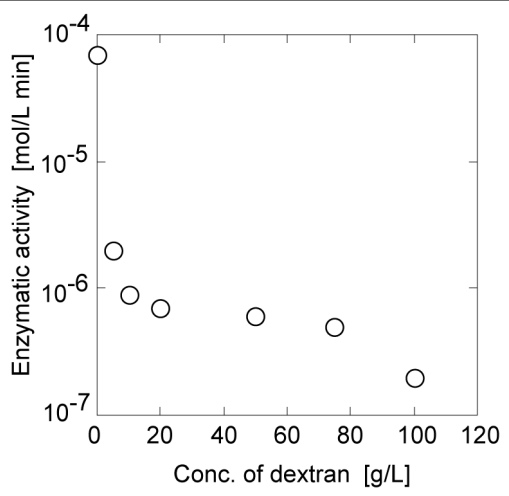

Figure 2. Viscosity of dextran solution. Temperature: $303 \mathrm{~K}$.

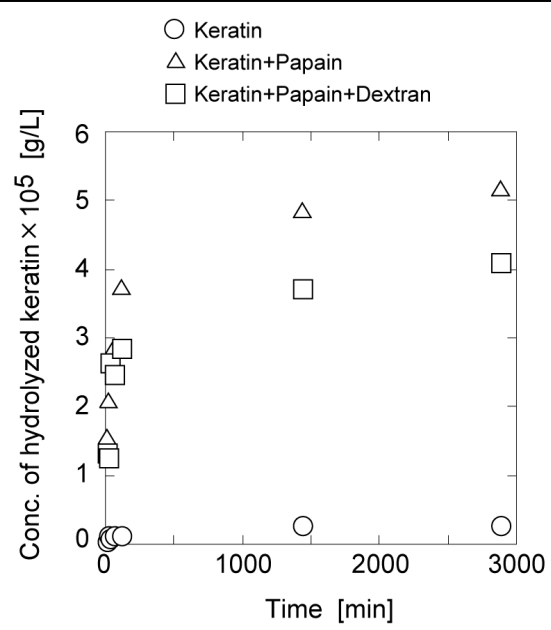

Figure 3. Hydrolysis of keratin powder by papain in dextran solution. The concentration of dextran: $50 \mathrm{~g} / \mathrm{L}$.

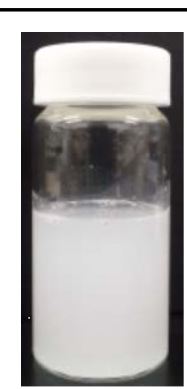

(a)

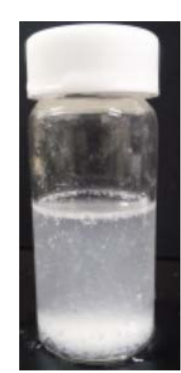

(b)

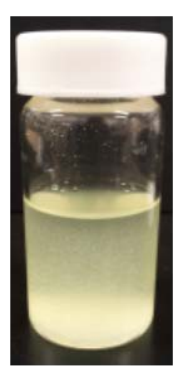

(c)

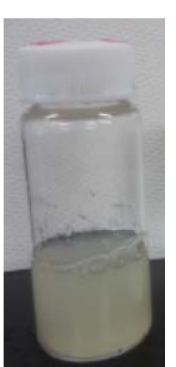

(d)

Figure 4. Images of keratin powder hydrolyzed by papain in dextran solution from Figure 3. (a) $t=0$, (b) $t=1440 \mathrm{~min}$, keratin + papain, (c) $\mathrm{t}=2880 \mathrm{~min}$, keratin+papain, (d) $\mathrm{t}=2880 \mathrm{~min}$, keratin + papain + dextran.

and dextran solutions, coloring the solution to yellow. Therefore, from Figure 4, due to no hydrolysis in only keratin and water solution, the solution remained white turbidity. The degree of yellow color in water was lower than that in dextran solution. In dextran solution, dextran is adsorbed to the surface of keratin to prohibit the hydrolysis by papain. 


\section{Final Remarks}

Papain activity in high concentration of dextran solution was determined for the peeling in cosmetics. Dextran has the plural hydroxyl groups and high solubility in water to show the high viscosity. With increasing the concentration of dextran, the activity of papain gradually increased. This was because the mobility of substrate and enzyme in dextran solution was low. Keratin, peel composition, was hydrolyzed by papain in dextran solution, demonstrating that papain in dextran solution has the potential composition for cosmetics.

\section{References}

[1] Song, J.Y., Kang, H.A, Kim, M.Y., Park, Y.M. and Kim, H.O. (2004) Damage and Recovery of Skin Barrier Function after Glycolic Acid Chemical Peeling and Crystal Microdermabrasion. Dermatologic Surgery, 30, 390-394. http://dx.doi.org/10.1046/j.1076-0512.2003.30107.x

[2] Berthold, J., Olsson, R.J.O. and Salmén, L. (1998) Water Sorption to Hydroxyl and Carboxylic Acid Groups in Carboxymethyl Cellulose (CMC) Studied with NIR-Spectroscopy. Cellulose, 5, 281-298. http://dx.doi.org/10.1023/A:1009298907734

[3] Hodge, R.M., Edward, G.H. and Simon, G.P. (1996) Water Absorption and States of Water in Semicrystalline Poly (Vinyl Alcohol) Films. Polymer, 37, 1371-1376. http://dx.doi.org/10.1016/0032-3861(96)81134-7

[4] Ashie, I.N.A., Sorensen, T.L. and Nielsen, P.M. (2002) Effects of Papain and a Microbial Enzyme on Meat Proteins and Beef Tenderness. Journal of Food Science, 67, 2138-2142. http://dx.doi.org/10.1111/j.1365-2621.2002.tb09516.X

[5] Sim, Y.C., Lee, S.G., Lee, D.C., Kang, B.Y., Park, K.M., Lee, J.Y., Kim, M.S., Chang, I.S. and Rhee, J.S. (2000) Stabilization of Papain and Lysozyme for Application to Cosmetic Products. Biotechnology Letters, 22, 137-140. http://dx.doi.org/10.1023/A:1005670323912

[6] Naessens, M., Cerdobbel, A., Soetaert, W. and Vandamme, E.J. (2005) Leuconostoc Dextransucrase and Dextran: Production, Properties and Applications. Journal of Chemical Technology and Biotechnology, 80, 845-860. http://dx.doi.org/10.1002/jctb.1322

[7] Casadei, M.A., Cerreto, F., Cesa, S., Giannuzzo, M., Feeney, M., Marianecci, C. and Paolicelli, P. (2006) Solid Lipid Nanoparticles Incorporated in Dextran Hydrogels: A New Drug Delivery System for Oral Formulations. International Journal of Pharmaceutics, 325, 140-146. http://dx.doi.org/10.1016/j.ijpharm.2006.06.012

[8] Lars, H. and Helle, B. (1995) Dextran Hydrogels for Colon-Specific Drug Delivery. Journal of Controlled Release, 36, 159-166. http://dx.doi.org/10.1016/0168-3659(95)00049-E

[9] Norlén, L. (2006) Stratum Corneum Keratin Structure, Function and Formation-A Comprehensive Review. International Journal of Cosmetic Science, 28, 397-425. http://dx.doi.org/10.1111/j.1467-2494.2006.00345.x

[10] Sitnitsky, A.E. (2008) Solvent Viscosity Dependence for Enzymatic Reactions. Physica A, 387, 5483-5497. http://dx.doi.org/10.1016/j.physa.2008.06.025 